\title{
A BAC-based physical map of the Drosophila buzzatii genome
}

\author{
Josefa González, ${ }^{1}$ Michael Nefedov, ${ }^{2}$ Ian Bosdet, ${ }^{3}$ Ferran Casals, ${ }^{1,5}$ Oriol Calvete, ${ }^{1}$ \\ Alejandra Delprat, ${ }^{1}$ Heesun Shin, ${ }^{3}$ Readman Chiu, ${ }^{3}$ Carrie Mathewson, ${ }^{3}$ Natasja Wye, ${ }^{3}$ \\ Roger A. Hoskins, ${ }^{4}$ Jacqueline E. Schein, ${ }^{3}$ Pieter de Jong, ${ }^{2}$ and Alfredo Ruiz ${ }^{1,2,6}$ \\ ${ }^{1}$ Departament de Genètica i de Microbiologia, Universitat Autònoma de Barcelona, 08193 Bellaterra (Barcelona), Spain; \\ ${ }^{2}$ Children's Hospital Oakland Research Institute, Oakland, California 94609, USA; ${ }^{3}$ Genome Sciences Centre, B.C. Cancer \\ Research Centre, Vancouver, Canada V5Z-4E6; ${ }^{4}$ Lawrence Berkeley National Laboratory, Berkeley, California 94720, USA
}

\begin{abstract}
Large-insert genomic libraries facilitate cloning of large genomic regions, allow the construction of clone-based physical maps, and provide useful resources for sequencing entire genomes. Drosophila buzzatii is a representative species of the repleta group in the Drosophila subgenus, which is being widely used as a model in studies of genome evolution, ecological adaptation, and speciation. We constructed a Bacterial Artificial Chromosome (BAC) genomic library of $D$. buzzatii using the shuttle vector pTARBAC2.1. The library comprises 18,353 clones with an average insert size of $152 \mathrm{~kb}$ and an $\sim 18 \times$ expected representation of the D. buzzatii euchromatic genome. We screened the entire library with six euchromatic gene probes and estimated the actual genome representation to be $\sim 23 \times$. In addition, we fingerprinted by restriction digestion and agarose gel electrophoresis a sample of 9555 clones, and assembled them using FingerPrint Contigs (FPC) software and manual editing into 345 contigs (mean of 26 clones per contig) and 670 singletons. Finally, we anchored 181 large contigs (containing 7788 clones) to the D. buzzatii salivary gland polytene chromosomes by in situ hybridization of 427 representative clones. The BAC library and a database with all the information regarding the high coverage BAC-based physical map described in this paper are available to the research community.
\end{abstract}

[Supplemental material is available online at www.genome.org. The following individuals kindly provided reagents, samples, or unpublished information as indicated in the paper: S. Celniker, B. Negre, and B. Pfeiffer.]

A variety of genomic resources have been developed as part of the Drosophila Genome Project, including the high-quality sequence and annotation of the Drosophila melanogaster genome (Adams et al. 2000; Celniker and Rubin 2003). Comparatively few genomic resources have been available for other species within the genus Drosophila. Phylogenetic analyses indicate that two main lineages exist within the Drosophila genus, which diverged $\sim 60$ million years ago (Powell 1997; Tamura et al. 2004). One lineage leads to the Sophophora subgenus with $\sim 300$ species (including $D$. melanogaster and D. pseudoobscura), whereas the other one leads to the subgenera Drosophila (including Drosophila virilis and Drosophila buzzatii) and Idiomyia (Hawaiian species), with $\sim 700$ and 375 described species, respectively (Powell 1997; http:// taxodros.unizh.ch/). Thus, many Drosophila species are relatively distantly related to $D$. melanogaster, and genomic resources developed for this species therefore have a somewhat limited applicability to them (Segarra et al. 1995; Podemski et al. 2001; Ranz et al. 2001; González et al. 2002). Fosmid and BAC libraries for some Drosophila species have been produced or are currently in production (http://www.genome.gov/; http://tdgc.arl.arizona. edu/baclibraries.htm). Recently, the genome sequence of Drosophila pseudoobscura became available (Richards et al. 2005), and

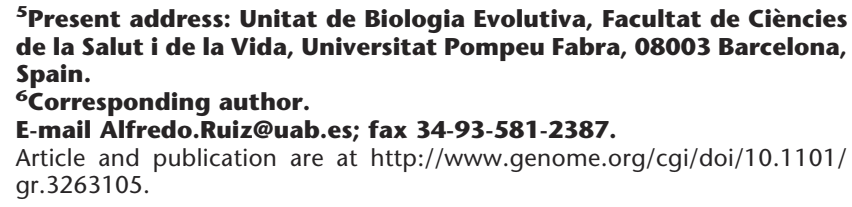

whole-genome shotgun sequences of 10 other Drosophila species are available or in progress (http://rana.lbl.gov/drosophila/ multipleflies.html).

Here, we describe the construction of a BAC library and a BAC-based physical map of the D. buzzatii genome. D. buzzatii belongs to the repleta species group of the Drosophila subgenus (Wasserman 1992), a group comprising 100 species that has been used for studies of ecological adaptation and speciation for more than 60 years (Spencer 1941; Crow 1942; Wharton 1942; Barker and Starmer 1982; Barker et al. 1990). Efforts to map the genome of $D$. buzzatii began 50 years ago with the comparative analysis of its salivary gland chromosomes to establish the phylogenetic relationships between repleta group species (Wasserman 1954, 1962; Ruiz et al. 1982; Ruiz and Wasserman 1993). This was followed by the linkage mapping of a small number of visible mutants (Schafer et al. 1993). In the last 10 years, $\sim 400$ DNA markers have been mapped by in situ hybridization to the D. buzzatii chromosomes (Ranz et al. 1997, 2003; Laayouni et al. 2000; Casals et al. 2003). No large-insert genomic libraries or clone-based physical maps were previously available for this species.

\section{Results and Discussion}

\section{Construction of $D$. buzzatii BAC library}

We constructed a BAC library from the D. buzzatii st-1 strain. High molecular-weight (HMW) DNA was prepared from adults, partially digested with EcoRI and EcoRI methylase, size frac- 
A
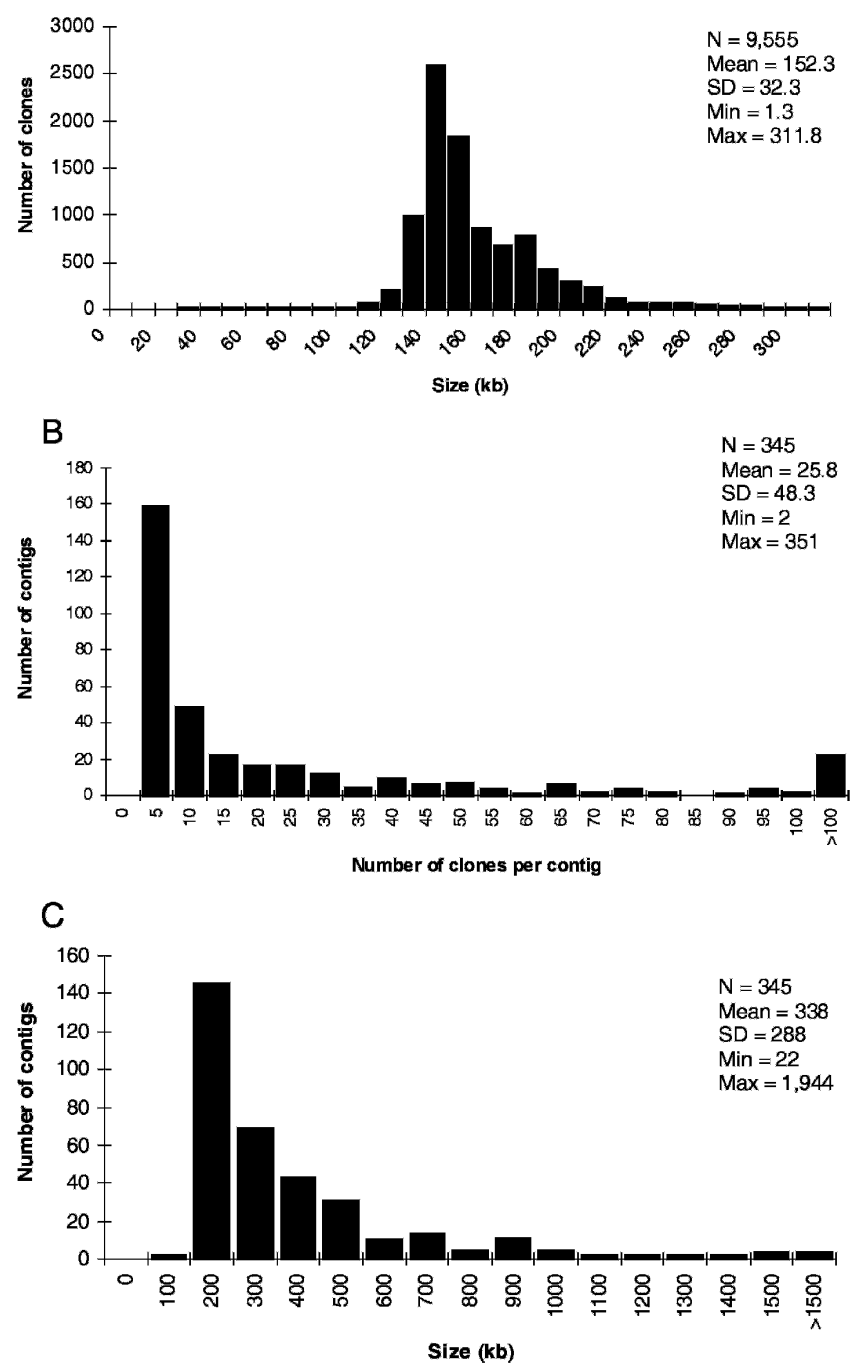

Figure 1. (A) Size distribution of the 9555 Drosophila buzzatii BAC clones analyzed by fingerprinting. (B) Distribution of clones in contigs and (C) contig sizes for the 345 contigs in the fingerprint map.

tioned, and cloned into the pTARBAC2.1 shuttle vector (Hoskins et al. 2000; Osoegawa et al. 2004). The D. buzzatii BAC library comprises 18,353 clones arrayed in 48 microtiter plates (see Methods). We determined the average insert size to be $152 \mathrm{~kb}$, by EcoRI restriction fingerprinting of 9555 clones (Fig. 1A). The size distribution is somewhat skewed to the right, which results in a very high proportion (98.6\%) of cloned inserts larger than 100 $\mathrm{kb}$. The size of the genomes of the repleta group species is $\sim 220$ $\mathrm{Mb}$, with $\sim 70 \%$ in the euchromatin (Ranz et al. 2001), thus the expected redundancy of the library is $\sim 18 \times$. We hybridized two gridded filters containing the entire library with six euchromatic gene probes. The average number of positive clones per probe was 23, which provides an estimate of the actual representation of the euchromatin in the library (see Supplemental material).

Fingerprinting and automatic contig assembly

To build a physical map of the D. buzzatii genome, we first fingerprinted and assembled into contigs 9555 BAC clones using high-throughput methods (Marra et al. 1997; Schein et al. 2004). The fingerprint data were automatically assembled using FingerPrint Contigs (FPC) software (Soderlund et al. 1997, 2000) with a cut-off score of $10^{-11}$. This threshold value represents the maximum allowable probability of a chance match between any two clones. The automated assembly produced 634 contigs and 516 unmatched clones (i.e., singletons, see Supplemental material for further details).

\section{Hybridization of BAC clones to salivary gland chromosomes}

We hybridized to the D. buzzatii chromosomes 552 clones representing 443 contigs. The information from 427 clones giving one primary hybridization signal was used for map construction. We also hybridized a subset of 163 BAC clones to the chromosomes of Drosophila repleta, another species of the repleta group whose cytological maps (Wharton 1942) have been used as the standard reference for all species in this group (Wasserman 1992). The results allowed us to revise the homology between chromosomes and chromosomal segments of D. buzzatii and D. repleta (Ruiz and Wasserman 1993) and to reconstruct the $D$. buzzatii chromosomes using the $D$. repleta cytological maps (Wharton 1942).

\section{An integrated physical map of the D. buzzatii genome}

Information from the fingerprint assembly, the cytological localization of BACs, and the library screening with gene probes was merged to produce an integrated physical map (see Methods). Manual editing and merging allowed us to reduce the number of contigs from the initial set of 634 to a final set of 345 . Figure 1B shows the distribution of clones within contigs. The mean number of clones per contig is 26 , and the largest number of clones in a contig is 351 . The fingerprints of a subset of overlapping clones within each contig were compared, and the size of the genomic region covered by each contig was estimated. The average contig size is estimated to be $338 \mathrm{~kb}$ (Fig. 1C). Some of the contigs are quite large (30 contigs are larger than $800 \mathrm{~kb}$ ), although many (216) are relatively small (100-300 kb). The largest contig is $\sim 1.9$ $\mathrm{Mb}$.

Using the cytological data, we anchored 181 contigs to the D. buzzatii chromosomes. These contigs contain 7788 (81.5\%) of the fingerprinted clones (Supplemental Table S1). Maps of the $D$. buzzatii chromosomes with the cytological localization of the 427 markers and the 181 contigs they represent are shown in Figure 2. A complete list of clones and in situ hybridization results is given in Supplemental Table S2.

The size and cytological span of 15 of the largest contigs were used to estimate the DNA content per cytological band in the salivary gland chromosome map. Taking into account the total number of bands and the total size of the contigs included in our integrated map (Fig. 2), we estimate that the physical map covers $\sim 89 \%$ of the euchromatic portion of the D. buzzatii chromosomes. The cytological data indicate that BAC coverage extends nearly to the telomeres, while pericentric regions are less well represented, probably because of the high content of repetitive DNA in these regions (Fig. 2).

Unrestricted access to the resources described in this paper is provided. A database containing all of the fingerprint images and analyses, clone sizes, contig composition, library screenings, and in situ hybridization images can be accessed using iCE (Fjell et al. 2003) at http://www.bcgsc.ca/ice. The D. buzzatii BAC library (CHORI-225) is available from BACPAC Resources (http://

\section{Genome Research} www.genome.org 


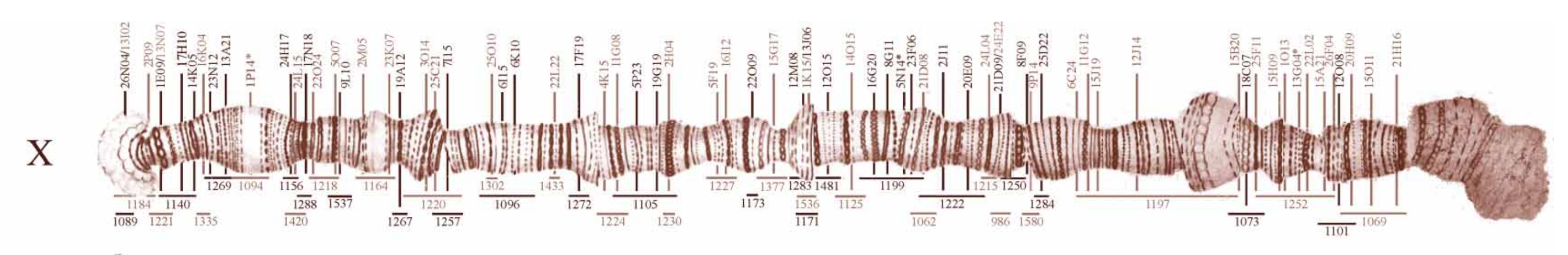

2

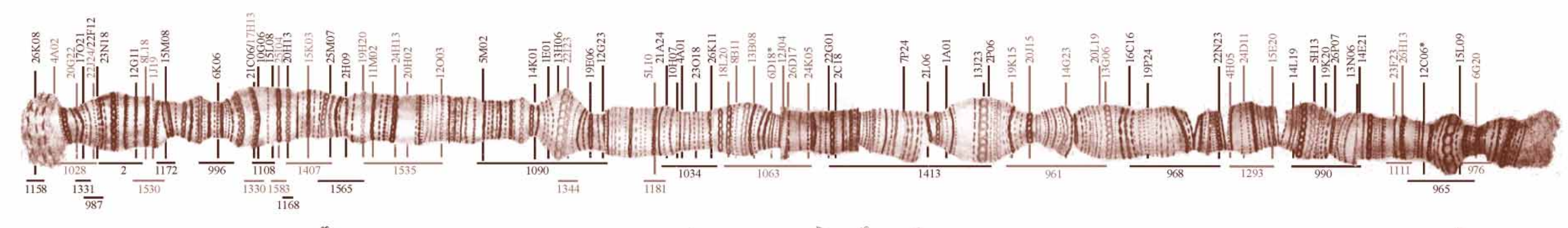

3

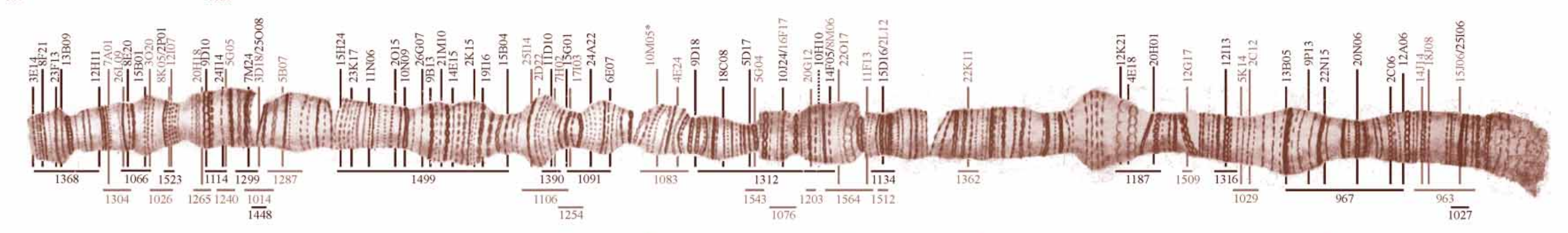
$6 \underset{110}{\frac{110}{180}}$

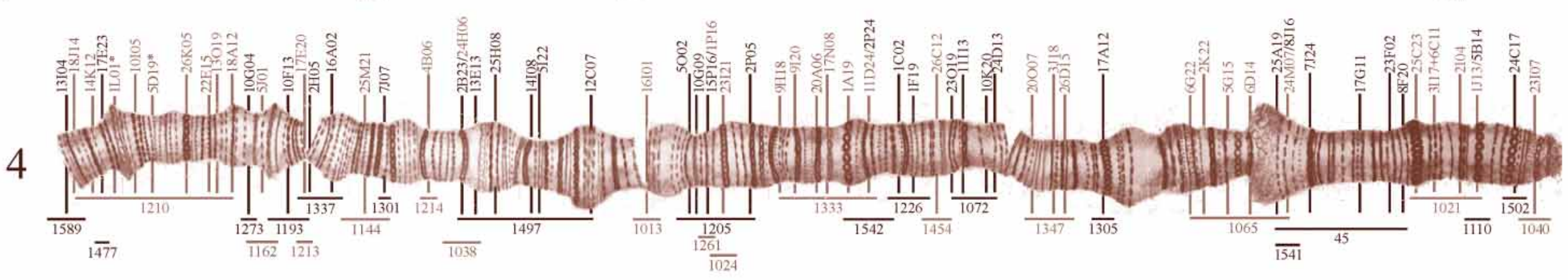
5 $\frac{1}{101}$ 
bacpac.chori.org/). We expect that the BAC library and highcoverage BAC-based physical map will be highly useful resources not only for those working in D. buzzatii as a model system but also to all those interested in the comparative analysis of genomes. The usefulness of this BAC-based physical map extends to many repleta group species, because their cytological relationships have been determined using $D$. repleta chromosomes as a reference (Wasserman 1992). In addition, the library has already been used to successfully sequence part of the Hox gene complex of D. buzzatii (Negre et al. 2005). Finally, the D. buzzatii map may help in the assembly of some of the Drosophila genomes currently being sequenced, particularly that of Drosophila mojavensis, which also belongs to the repleta species group.

\section{Methods}

\section{Flies}

The D. buzzatii strain used to construct the BAC library and to map BACs by in situ hybridization (st-1) is fixed for the standard arrangement in all chromosomes and was produced by Betrán et al. (1998). The D. repleta stock used for in situ hybridization was no. 1611.6 from the National Drosophila Species Resource Center (Bowling Green, $\mathrm{OH}$ ).

\section{BAC library construction}

The library was constructed according to the improved methods described in detail in Frengen et al. (1999) and Osoegawa et al. $(1999,2004)$. HMW DNA was prepared from $3 \mathrm{~g}$ of adult flies, including equal numbers of females and males, as described in Hoskins et al. (2000). The partially digested HMW DNA was sizefractionated by Pulse Field Gel Electrophoresis, and fractions corresponding to $150-250-k b$ DNA fragments were recovered by electroelution and cloned in pTARBAR2.1. See Supplemental material for further details.

\section{In situ hybridization of BAC clones}

In situ hybridizations were carried out as in González et al. (2002). Probes were labeled with biotin-16-dUTP. The hybridization temperatures were $37^{\circ}$ for $D$. buzzatii chromosomes and $25^{\circ}$ for $D$. repleta chromosomes. We hybridized 552 BAC clones to $D$. buzzatii salivary gland chromosomes; 506 gave positive results, and 427 producing a single primary hybridization signal were used in physical map construction (Supplemental Tables S1 and S2). Nine clones gave two signals; these may represent chimeric clones or mixtures of two clones caused by cross-well contamination. This low rate (1.6\%) is in agreement with the low level of chimerism observed in other BAC libraries (Osoegawa et al. 2001). In total, 70 clones gave more than two hybridization signals and/or hybridized to the pericentromeric regions and the microchromosome, probably because of repetitive DNA content. The density of transposable elements increases near Drosophila centric heterochromatin (Kaminker et al. 2002). Clones from such repeat-rich regions cannot be assigned to a particular chromosomal site. Thus, there is a relative scarcity of markers in our physical map near the centric heterochromatin, especially on the X-chromosome (Fig. 2). Examples of the different types of hybridization results are shown in Supplemental Figure S1.

\section{Acknowledgments}

We thank Susan Celniker, Bàrbara Negre, Barret Pfeiffer, Theresa Ren, and Chung Li Shu for help. This work was supported by grant BMC2002-01708 from the Dirección General de Enseñanza Superior e Investigación Científica (MEC, Spain) awarded to A.R.

\section{References}

Adams, M.D., Celniker, S.E., Holt, R.A., Evans, C.A., Gocayne, J.D., Amanatides, P.G., Scherer, S.E., Li, P.W., Hoskins, R.A., Galle, R.F., et al. 2000. The genome sequence of Drosophila melanogaster. Science 287: 2185-2195.

Barker, J.S.F. and Starmer, W.T., eds. 1982. The cactus-yeast-Drosophila model system. Academic Press, Sydney, Australia.

Barker, J.S.F., Starmer, W.T., and MacIntyre, R., eds. 1990. Ecological and evolutionary genetics of Drosophila. Plenum Press, New York.

Betrán, E., Santos, M., and Ruiz, A. 1998. Antagonistic pleiotropic effect of second-chromosome inversions on body size and early life-history traits in Drosophila buzzatii. Evolution 52: 144-154.

Casals, F., Cáceres, M., and Ruiz, A. 2003. The Foldback-like transposon Galileo is involved in the generation of two different natural chromosomal inversions of Drosophila buzzatii. Mol. Biol. Evol. 20: $674-685$.

Celniker, S.E. and Rubin, G.M. 2003. The Drosophila melanogaster genome. Annu. Rev. Genomics Hum. Genet. 4: 89-117.

Crow, J.F. 1942. Cross fertility and isolating mechanisms in the Drosophila mulleri group. Univ. Texas Publ. 4228: 53-67.

Fjell, C.D., Bosdet, I., Schein, J.E., Jones, S.J., and Marra, M.A. 2003. Internet Contig Explorer (iCE) a tool for visualizing clone fingerprint maps. Genome Res. 13: 1244-1249.

Frengen, E., Weichenhan, D., Zhao, B., Osoegawa, K., van Geel, M., and de Jong, P. 1999. A modular, positive selection bacterial artificial chromosome vector with multiple cloning sites. Genomics 58: $250-253$.

González, J., Ranz, J.M., and Ruiz, A. 2002. Chromosomal elements evolve at different rates in the Drosophila genome. Genetics 161: $1137-1154$.

Hoskins, R.A., Nelson, C.R., Berman, B.P., Laverty, T.R., George, R.A., Ciesiolka, L., Naeemuddin, M., Arenson, A.D., Durvin, J., David, R.G., et al. 2000. A BAC-based physical map of the major autosomes of Drosophila melanogaster. Science 287: 2271-2274.

Kaminker, J.S., Bergman, C.M., Kronmiller, B., Carlson, J., Svirskas, R., Patel, S., Frise, E., Wheeler, D.A., Lewis, S.E., Rubin, G.M., et al. 2002. The transposable elements of the Drosophila melanogaster euchromatin: A genomic perspective. Genome Biol. 3: research0084.

Laayouni, H., Santos, M., and Fontdevila, A. 2000. Toward a physical map of Drosophila buzzatii: Use of randomly amplified polymorphic DNA polymorphisms and sequence-tagged site landmarks. Genetics 156: $1797-1816$.

Marra, M.A., Kucaba, T.A., Dietrich, N.L., Green, E.D., Brownstein, B., Wilson, R.K., McDonald, K.M., Hillier, L.W., McPherson, J.D., and Waterson, R.H. 1997. High throughput fingerprint analysis of large-insert clones. Genome Res. 7: 1072-1084.

Negre, B., Casillas, S., Suzanne, M., Sánchez-Herrero, E., Akam, M. Nefedov, M., Barbadilla, A., de Jong, P., and Ruiz, A. 2005. Conservation of regulatory sequences and gene expression patterns in the disintegrating Drosophila Hox gene complex. Genome Res. 15: 692-700.

Osoegawa, K., de Jong, P., Frengen, E., and Ioannou, P.A. 1999. Construction of bacterial artificial chromosome (BAC) libraries. In Current protocols in human genetics (eds. N.C. Dracopoli et al.), pp. 5.15.1-5.15.33. Wiley, New York.

Osoegawa, K., Mammoser, A.G., Wu, C., Frengen, E., Zeng, C., Catanese, J.J., and de Jong, P.J. 2001. A bacterial artificial chromosome library for sequencing the complete human genome. Genome Res. 11: $483-496$.

Osoegawa, K., Zhu, B., Shu, C.L., Ren, T., Cao, Q., Vessere, G.M., Lutz, M.M., Jensen-Seaman, M.I., Zhao, S., and de Jong, P.J. 2004. BAC resources for the rat genome project. Genome Res. 14: 780-785.

Podemski, L., Ferrer, C., and Locke, J. 2001. Whole arm inversions of chromosome 4 in Drosophila species. Chromosoma 110: 305-312.

Powell, J.R. 1997. Progress and prospects in evolutionary biology. The Drosophila model. Oxford University Press, New York.

Ranz, J.M., Segarra, C., and Ruiz, A. 1997. Chromosomal homology and molecular organization of Muller's elements D and $\mathrm{E}$ in the Drosophila repleta group. Genetics 145: 281-295.

Ranz, J.M., Casals, F., and Ruiz, A. 2001. How malleable is the eukaryotic genome? Extreme rate of chromosomal rearrangements in the genus Drosophila. Genome Res. 11: 230-239.

Ranz, J.M., González, J., Casals, F., and Ruiz, A. 2003. Low occurrence of gene transposition events during the evolution of the genus Drosophila. Evolution 57: 1325-1335. 


\section{González et al.}

Richards, S., Liu, Y., Bettencourt, B.R., Hradecky, P., Letovsky, S., Nielsen, R., Thornton, K., Hubisz, M.J., Chen, R., Meisel, R.P., et al. 2005. Comparative genome sequencing of Drosophila pseudoobscura: Chromosomal, gene, and cis-element evolution. Genome Res. 15: $1-18$.

Ruiz, A. and Wasserman, M. 1993. Evolutionary cytogenetics of the Drosophila buzzatii species complex. Heredity 70: 582-596.

Ruiz, A., Fontdevila, A., and Wasserman, M. 1982. The evolutionary history of Drosophila buzzatii. III. Cytogenetic relationships between two species of the buzzatii cluster. Genetics 101: 503-518.

Schafer, D.J., Fredline, D.K., Knibbs, W.R., Green, M.M., and Barker, J.S.F. 1993. Genetics and linkage mapping of Drosophila buzzatii. J. Hered. 84: 188-194.

Schein, J., Kucaba, T., Sekhon, M., Smailus, D., Waterston, R., and Marra, M. 2004. High-throughput BAC fingerprinting. In Methods in molecular biology (eds. S. Zhao and M. Stodolsky), Vol. 255, pp. 143-156. Humana Press, Totowa, NJ.

Segarra, C., Lozovskaya, E.R., Ribó, G., Aguadé, M., and Hartl, D.L. 1995 P1 clones from Drosophila melanogaster as markers to study the chromosomal evolution of Muller's A element in two species of the obscura group of Drosophila. Chromosoma 104: 129-136.

Soderlund, C., Longden, I., and Mott, R. 1997. FPC: A system for building contigs from restriction fingerprinted clones. Comput. Appl. Biosci. 13: 523-535.

Soderlund, C., Humphray, S., Dunham, A., and French, L. 2000. Contigs built with fingerprints, markers, and FPC V4.7. Genome Res. 10: $1772-1787$.

Spencer, W.P. 1941. Ecological factors and Drosophila speciation. Ohio J. Sci. 41: $190-200$

Tamura, K., Subramanian, S., and Kumar, S. 2004. Temporal patterns of fruit fly (Drosophila) evolution revealed by mutation clocks. Mol.
Biol. Evol. 21: 36-44.

Wasserman, M. 1954. Cytological studies of the repleta group. Univ. Texas Publ. 5422: 130-152.

- 1962. Cytological studies of the repleta group of the genus

Drosophila. V. The mulleri subgroup. Univ. Texas Publ. 6205: 85-117.

. 1992. Cytological evolution of the Drosophila repleta species

group. In Drosophila inversion polymorphism (eds. C.B. Krimbas and J.R. Powell), pp. 455-552. CRC Press, Boca Raton, FL.

Wharton, L.T. 1942. Analysis of the repleta group of Drosophila. Univ.

Texas Publ. 4228: 23-52.

\section{Web site references}

http://bacpac.chori.org/; BACPAC Resources Center at Children's Hospital Oakland Research Institute.

http://rana.lbl.gov/drosophila/multipleflies.html; Assembly/Alignment/Annotation of 12 Drosophila Genomes.

http://taxodros.unizh.ch/; The database on Taxonomy of Drosophilidae.

http://tdgc.arl.arizona.edu/baclibraries.htm; Tucson Drosophila Genomics Consortium.

http://www.bcgsc.ca/ice; Internet Contig Explorer (iCE) download from the Genome Sciences Centre (British Columbia Cancer Agency) server.

http://www.genome.gov/; National Human Genome Research Institute.

Received September 16, 2004; accepted in revised form March 22, 2005.

\section{Genome Research}

www.genome.org 


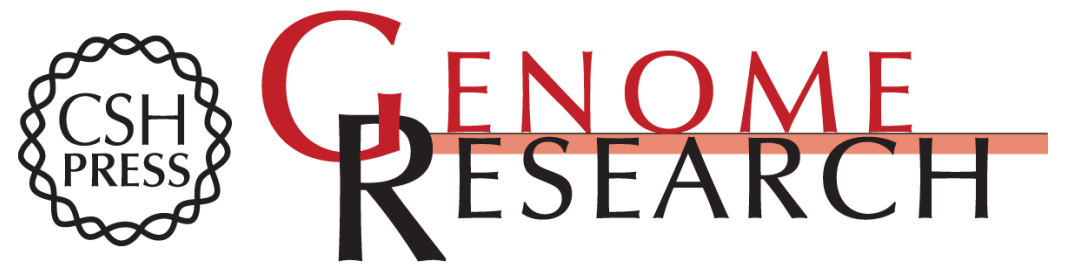

\section{A BAC-based physical map of the Drosophila buzzatii genome}

Josefa González, Michael Nefedov, Ian Bosdet, et al.

Genome Res. 2005 15: 885-892

Access the most recent version at doi:10.1101/gr.3263105

Supplemental
Material http://genome.cshlp.org/content/suppl/2005/05/18/15.6.885.DC1

References This article cites 32 articles, 14 of which can be accessed free at: http://genome.cshlp.org/content/15/6/885.full.html\#ref-list-1

\section{License}

Email Alerting Service top right corner of the article or click here.

\section{Affordable, Accurate Sequencing.}

